tumeur $2,2 \mathrm{~cm}$. La tumeur était rigide, de consistance cartilagineuse et par conséquent inextensible; comme elle enveloppait presque complètement l'estomac, celui-ci ne pouvait pas se distendre et augmenter sa capacité par l'apport de la nourriture. Ce viscère présentait l'aspect que l'on constate lors de l'anorexie de reproduction, c'est-à-dire que ses parois sont

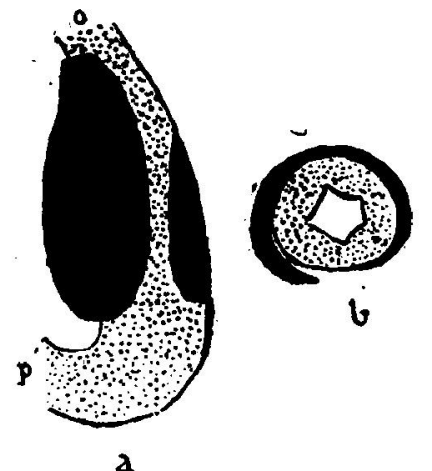

FiG. 13. - Myome chez un Cortgone.

1. L'estomac et la tumeur. vus de champ;

2. Coupe transversale de l'estomac et de la tumeur; $-O$, région terminale de P'cosophake; - $P$, région précédent le pylore.

La tumeur est en noir et l'estomac en pointilié.

très épaissies et sa capacité fort limitée. Il cétait bourré de débris de squelettes de petits poissons.

Dans l'espèce humaine, ces tumeurs sont beaucoup plus fréquentes chez les individus âgés que chéz les jeunes et il est vraisemblable que, dans le cas en question, il y ait une relation entre la genèse du myome et l'âge de la Palée. Ajoutons que, chez celle-ci, le tissu graisseux entourant les viscères abdominaux était développé d'une façon extraordinaire et que les testicules étaient réduits à deux minces rubans ; l'individu était certainement stérile, soit à cause de son grand âge, soit par le fait que ses glandes génitales étaient toujours restées atrophiées.

\title{
L'ÉQUIPEMENT PISGIGOLE DU BASSIN DE L'ADOUR
}

\author{
Par M. J. LARRIEU \\ Inspecteur principal des Eaux et Foréts, aloron.
}

L'Adour, fleuve côtier du versant Atlantique, arrose soit directẹment, soit par ses affluents, trois départements de la région du Sud-Ouest : les Hautes-Pyrénées, les Landes et les Basses-Pyrénées (Fig. 44). 
Ce bassin, d'une étendue relativement faible, présente, du point de vue piscicole, un très grand intérêt : il comprend en effet de magnifiques zones à Truite dans la montagne et basse montagne; des zones à Cyprinides dans la plaine et, par-dessus tout, il est remarquable par la présence de nombreux migrateurs : Muges, Lamproie, Anguille, Alose et Saumon. Cette

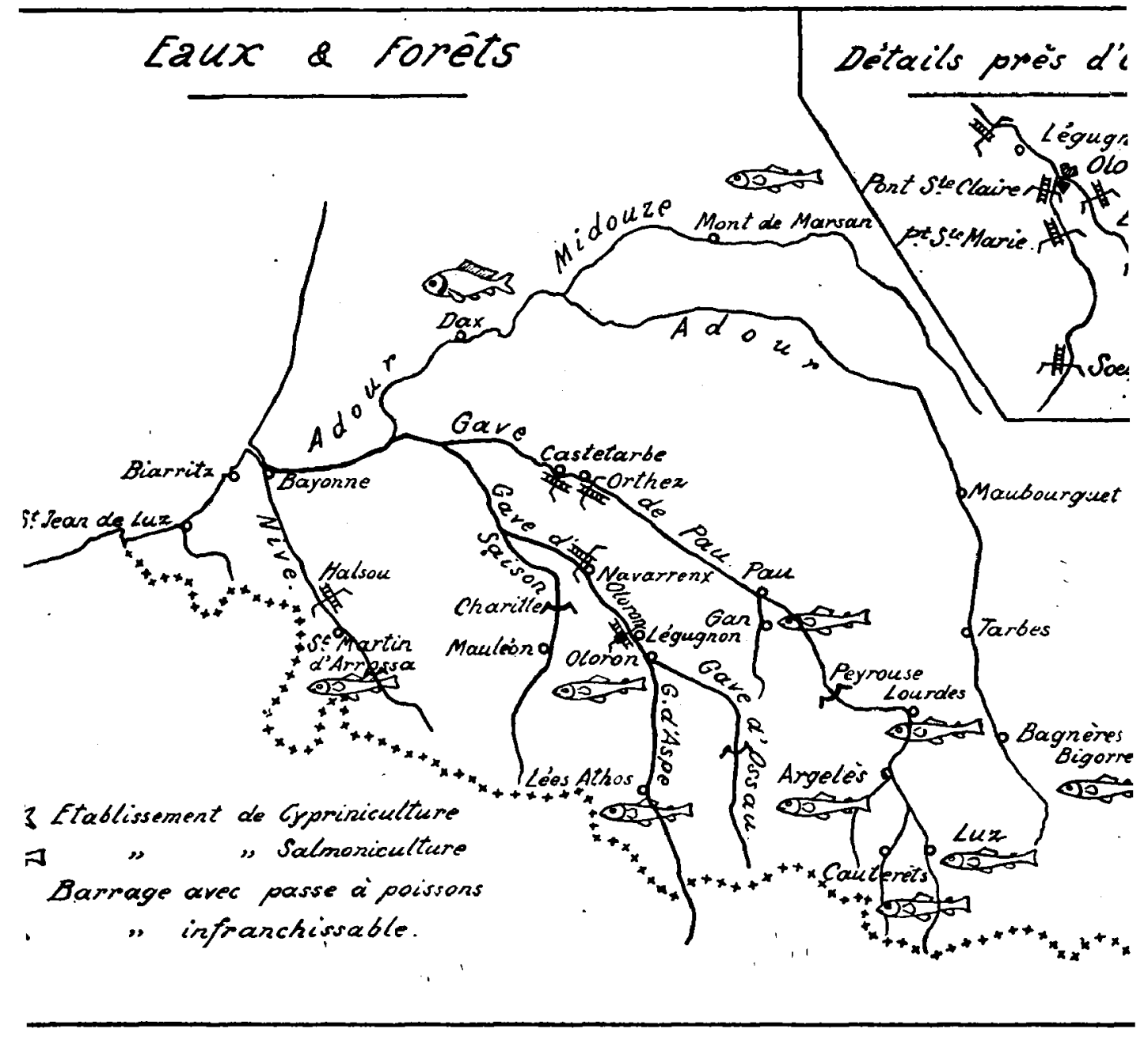

Fig. 44. - Carte du Bussin de l'Adour.

dernière espèce s'était naturellement maintenue abondante jusqu'à ces dernières années dans le Gave d'Oloron et la Nive, tandis qu'elle avait disparu à peu près complètement de l'Adour supérieur, du Gave de Mauléon et du Gave de Pau.

On comprendra facilement que la question du Saumon, qui est vraiment le roi de nos rivières, ait particulièrement intéressé les populations et les services administratifs du bassin de l'Adour. C'est qu'en effet $9^{\circ 0}$ familles de pècheurs tirent le plus clair de leur revenu de la pêche du Saumon. Le 
nombre de sujets capturés doit être, en moyenne, de to.000 par an, ce qui représente, tant pour les inscrits maritimes que pour les pêcheurs au lancer, un revenu de 4.000.000 de francs ( 400 'fr. par pièce en moyenne).

Mais ce chiffre ne représente que la valeur du poisson. capturé. Il faut tenir'compte, en outre, des richesses appoŕtées au pays par la clientèle étrangère, sportifs anglais en particulier, très attirés chez nous par la pêche du Saumon.

La disparition de ce poisson dans les bassins de la Seine et de la Garonne, à la suite de la construction de barrages infranchissables qui empêchent sa reproduction, a fait craindre que pareille mésaventure n'arrivât dans le bassin de l'Adour. C'est pourquoi des mesures de protection spéciales ont été édictées : - interdiction de construire de nouveaux barrages - et que des travaux importants ont été entrepris pour assurer la reproduction de l'espèce.

Dans l'Adour, en amont du confluent avec les gaves, le Saumon a dú disparaître depuis fort longtemps, car dans la zone des frayères, - région de Bagnères-de-Bigorre - Tarbes - Maubourguet, - la rivière est presque à sec chaque été, ses eaux servant à l'arrosage des prairies de la plaine de Tarbes. Les canaux d'irrigation datant de l'époque d'Aleric ( $v^{\circ}$ siècle), il est vraisemblable que la disparition du Saumon dans le Haut-Adour est très ancienne.

En ce qui concerne le Gave de Mauléon, l'arrêt des migtations était provoqué par le barrage d'Osserain situé à quelques kilomètres à peine du confluent avec le Gave d'Oloron. La rivière était de ce fait, considérée comme sans intérêt pour le Saumon.

Pax contre, jusqu'en 1917 le poisson était encore abondant dans le Gave de Pau. Mais, à cette époque, fut édifié en aval d'Orthèz le barrage de Castetarbe constituant un obstacle infranchissable. Pendant 4 ou 5 ans, jusque vers 1922, on put voir encore les géniteurs remonter jusqu'en ce point ; puis petit à petit, le Gave de Pau fut abandonné par'le Saumon qui ne pouvait plus atteindre ses frayères.

Le Gave de Pau ne recevánt plus de reproducteurs, le nombre des tacons descendant à la mer avait fortement diminué pour l'ensemble du bassin et, comme conséquence, les captures d'adultes par les inscrits maritimes accusaient une baisse très marquée.

L'Administration des Eaux et Forêts s'émut de cet état de chosas et, dès r922, sur la proposition de $M$. le Conservateur Crambzad, il fut décidé de créer, dans la $22^{\circ}$ Conservation, un établissement de pisciculture pour élever des alevins de Saumon qui seraient immergés dans la zone des frayères du Gave de Pau. Cet établissement, installé à Oloron, malgré certains défauts, a rempli son rôle et tous les ans, il est procédé dans le Gave de Pau à des déversements qui maintiennent dans ce cours d'eau un courant de tacons descendant à la mer. 
Mais on ne pouvait songer à repeupler entièrement le Gave de Pau par des déversements artificiels. Il fallait permettre aux géniteứrs Saumons de remonter à leurs frayères. Deux obstacles les en empêchaient : le barrage de Castetarbe d'abord, puis le barrage d'Orthez Ville.

En r928, une première échelle à poissons fut construite dans le barrage de Castetarbe. C'était une échelle genre DeniL avec amortisseurs en fonte. Malheureusement, la mise en place était à peine terminée qu'une crue extrêmement violente arracha les maçonneries dont la prise n'était pas encore faite.

Par ailleurs, deux échelles similaires avaient été construites, à la même époque, l'une à Orthez-Ville sur le Gave de Pau, l'autre sur la Nive au barrage d'Halsou qui venait d'être relevé.

Ces échelles n'ont pas paru donner les résultats escomptés.

Fntre temps, M. l'Inspecteur Général des Faux et Forêts de Lachadenedr. avait mis au point un système de passe à couloir beaucoup plus large que celui des échelles précédentes et équipée dans le fond d'amortisseurs en chevron (I). De son côté, M. Aveque, Ingénieur-Conseil à Pau, avait imaginé un mécanisme de fermeture automatique de la passe, pour éviter la perte d'eau pendant les périodes où le poisson reste cantonné dans les grands fonds. L'échelle de LACHADENEDE a, en effet, l'inconvénient d'absorber un débit de 1.000 à 1.200 litres-seconde, ce qui peut gêner l'usinier pendant la période d'étiage.

Mais, commé à cette époque le Saumon ne se déplace pas, la passe peut être fermée sans inconvénient. Le système automatique ouvrant le passage dès que l'eau affleure la crête du barrage, le poisson peut profiter des moindres crues pour franchir la chute.

Un modèle réduit d'échelle de ce système a figuré en ig3r à I'Exposition des Arts et Techniques de Paris.

La première construction de ce genre a été réalisée en i 932 sur la Nive au barrage d'Halsou. Les migrateurs remontèrent facilement la passe. Devant les résultats obtenus, il fut alors décidé d'équiper les gaves par la construction d'échelles système LACHADENEDE avec adjonction d'un clapet automatique système Aveque.

Sur ce type, dès r $9^{33}$ sont mises en chantier les échelles de Castetarbe et Orthez-Ville sur le Gave de Pau et celle de Légugnon sur le Gave d'Oloron. Celle de Navarrenx suit en r934, également sur le Gave d'Oloron.

Cependant, en 1936, les Gaves d'Aspe et d'Ossau, dont la réunion forme le Gave d'Oloron, étaient encore interdits au Saumon par cinq barrages infranchissables situés aux environs de la Ville d'Oloron.

La loi du 18 août i 936 sur les grands travaux contre le chômage allait permettre de remédier à ce facheux état de choses, en donnant aux géni-

(.) Voir au sujet de ces échelles : - Bulletin, $-\mathrm{n}^{\circ} 85$, Juillet 1935, p. $5 ;-\mathrm{n}^{\circ} 116$, Janvier-Février, 1939 , p. 97. 
teurs la possibilité de remonter jusqu'aux belles frayères qu'ils fréquentaient autrefois.

Au cours de l'année r 936 , 'deux échelles sont construites sur le Gave d'Aspe, aux Barrages de l'Usine BEDAT et de la Société Hydro-électrique en amont du pont de Sainte-Marie.

En $9_{9}^{3} 7$, deux autres sont réalisées, l'une sur le Gave d'Aspe (Usine de Soeix), l'autre sur le Gave d'Ossau (Usine Baraban).

Enfin, en r938, la seconde échelle du Gave d'Ossau (barrage Etchrbarne) est terminée et mise en service.

Ces travaux ont permis au Saumon de récupérer 28 kilomètres frayères sur le Gave d'Aspe et environ 22 kilomètres sur le Gave d'Ossau. Déjà, en 1938, des Saumons ont été vus et même capturés à to kilomètres en amont d'Oloron, en des points où ce poisson n'était plus revenu depuis plusieurs siècles.

Il est a remarquer que la Truite bénéficie également -de ce nouvel état de choses et, que les géniteurs de cette espèce utilisent largement les passes construites.

En ce qui concerne le Gave de Mauléon ou Saison, la nature s'est chargée de remettre la rivière en son état primitif, du moins dans sa partie moyenne. Une crue, survenue pendant la dernière guerre, démolit le barrage d'Osserain ouvrant à nouveau le passage du Saumon, qui depuis, est redevenu abondant dans le Saison.

Parallèlement à la construction des échelles, grâce encore à la loi du I 8 aout r 936 , l'Administration des Eaux et Forêts a édifié de toutes pièces ou amélioré six établissements de salmoniculture destinés à élever Saumon, Truites et aussi Saumon de Fontaine et Omble-Chevalier pour les régions élevées et, en particulier, pour les lacs de barrage à haute altitude, aingi qu'un Parc-frayère pour la zone à Cyprinides.

Ces'sept établissements, ajoutés aux deux établissements domaniaux déjà existants (Oloron et Luz-Saint-Sauveur) et aux trois stations d'alevinage des Sociétés de Pêche de Pau, d'Oloron et de Lourdes, donnent un total de douze installations d'élevage pour l'ensemble du bassin de l'Adour :

Ces installations se répartissent comme suit :

Sur le Gave d'Ossau. - Salmoniculture d'Oloron appartenant à la Société de Pêche d'Oloron.

Sur le Gave d'Aspe. - Salmoniculture de Lees-Athas, appartenant à l'Etat.

Sur le Gave d'Oloron. - Salmoniculture d'Oloron, appartenant à l'Etat.

Sur la Nive. - Salmoniculture de Saint-Martin-d'Arnossa, appartenant à l'Etat.

Sur l'Adour. - Salmoniculture de Bagnères-de-Bigorre, appartenant à I'Etat. 
Sur le Gave d'Azun (affluent du Gave de Pau). - Salmoniculture d'Argelès-Gazost, appartenant à l'Etat.

Sur le Gave de Cauterets (affluent du Gave de Pau). - Salmoniculture de Cauterets, appartenant à l'Etat.

Sur le Gave de Pau. - Salmoniculture de Lourdes, appartenant à la Société de Pêche de Lourdes.

Sur le Bastan (affluent du Gave de Pau). - Salmoniculture de Luz-SaintSauveur, appartenant à l'Etat.

Sur le Neez (affluent du Gave de Pau). - Salmoniculture de Gan, appartenant à la Société de Pêche de Pau.

Sur la Midouze. - Salmoniculture de Mont-de-Marsan, appartenant à l'Etat.

Sur l'Adour. - Cypriniculture de Dax, appartenant à l'Etat.

Cet ensemble peut traiter, au minimum, deux millions d'oufs de Salmonides dont une partie pourra être fournie par les géniteurs de Truites stabulés dans les bassins ou étangs aménagés à Lees-Athas, Oloron, Argelès et Cauterets. Les oufs de Saumon sont prélevés sur des géniteurs sauvages capturés au moment de la fraye.

Les établissements du bassin de l'Adour sont aujourd'hui terminés et pourront fonctionner à plein à partir de l'an prochain.

Leur production fournira un appoint très sérieux à la reproduction naturelle devenue insuffisante en raison du grand nombre de touristes qui s'adonnent au sport de la pêche dans toutes les stations thermales des Pyrénées.

A côté des établissements de production, qui constituent la partie pratique de l'ouvre, une part a été consacrée à la partie scientifique et grâce à l'obligeance de la Ville de Biarritz, qui a offert le terrain, un laboratoire a été créé au Musée de la Mer en vue d'étudier la biologie des poissons migrateurs qui fréquentent les cours d'eau de la région du Sud-Ouest (I).

A la sụite des travaux exécutés, on peut considérer que le bassin de l'Adour est aujourd'hui équipé de façon à réduire, dans une large mesure, les dommages piscicoles causés par la présence des barrages qui alimentent en force hydraulique les nombreuses usines de la région pyrénéenne, centre et ouest.

Toutefois, cet aménagement demanderait encore comme compléments la construction d'une échelle à Saumon sur la Nive au barrage d'Ossès, difficilement franchissable, et d'une seconde sur le Gave de Mauléon aú barrage de Charritte, tout à fait infranchissable.

Ce sera le travail de demain.

(1) Voir Bulletin: $-\mathrm{n}^{\circ}$ I 3 , Juillet-Août 1938 , p. 12. 